New Test. Stud. (2021), 67, pp. 134-156. () The Author(s), 2020. Published by Cambridge University Press. This is an Open Access article, distributed under the terms of the Creative Commons Attribution licence (http://creativecommons.org/ licenses/by/4.o/), which permits unrestricted re-use, distribution, and reproduction in any medium, provided the original work is properly cited.

doi:10.1017/S0028688520000144

\title{
Kritische Exegese. Der 1829 von H. A. W. Meyer begründete „Kritische und exegetische Kommentar über das Neue Testament" in forschungsgeschichtlicher Sicht
}

\author{
DIETRICH-ALEX KOCH \\ Westfälische Wilhelms-Universität Münster, Evangelisch-Theologische Fakultät, \\ Universitätsstraße 13-17, D-48143 Münster (W.), Germany. \\ Email:dakoch@uni-muenster.de
}

\begin{abstract}
The 'Kritisch-exegetischer Kommentar über das Neue Testament', formerly called 'The Meyer', was established more than 180 year ago. As the KEK is the oldest commentary series in German (and not only in German) still existing today, it is an important subject of research and the analysis of the development of the series reveals many significant insights in the history of New Testament scholarship in the last two centuries. Last but not least, recent investigations shed light on the relations between the German and the English-speaking scholarship in the nineteenth and twentieth centuries.
\end{abstract}

Keywords: 'Kritisch-exegetischer Kommentar', commentaries, H. A. W. Meyer, B. Weiß, R. Bultmann, E. Haenchen

1829 veröffentlichte Heinrich August Wilhelm Meyer, ein bis dahin völlig unbekannter evangelischer Dorfpfarrer aus dem Herzogtum SachsenMeiningen, mit 29 Jahren eine zweibändige Textausgabe des Neuen Testamentes mit paralleler deutscher Übersetzung. Zum Gesamtkonzept gehörte, wie das Titelblatt (Abb. 1) $)^{1}$ zeigt, von vornherein ein „kritischer und

1 Die Abbildung wurde entnommen aus: Der „Kritisch-exegetische Kommentar" in seiner Geschichte: H.A.W. Meyers KEK von seiner Gründung 1829 bis heute (hg. E.-M. Becker/F. W. Horn/D.-A. Koch; KEK Sonderband; Göttingen: Vandenhoeck \& Ruprecht, 2018) 534. Dieser erste Band („Abtheilung“) des Gesamtwerks umfasste XxxII (Vorwort) und 599 Seiten (Text und Übersetzung der vier Evangelien), der dazugehörige zweite Band mit 815 Seiten Text und Übersetzung der Apostelgeschichte, der neutestamentlichen Briefe und der Apokalypse; zusammen bildeten beide Bände („Abtheilungen“) den „Ersten Theil“. Den „Zweiten Theil“ bildeten dann die Kommentare. - In den ersten acht Jahrzehnten des KEK lautet die Verlagsangabe ausnahmslos „Vandenhoeck und Ruprecht“. Das sog. „kaufmännische und-Zeichen“ (\&) wurde vom Verlag erst im Laufe des ersten Jahrzehnts 


\section{D a s \\ NEUE TESTAMENT \\ Griech is c h}

nach den besten Hülfsmitteln kritisch revidirt

mit einer

neuen Deutschen Übersetzung

und einem

kritischen und exegetischen Kommentar

Heinr. Aug. Wilh. Meyer,

Pfarrer zu Osthausen bei Kranichfeld in Herzogthume Meiningeu.

\section{Erster Theil}

den Griechischen Text und dis Deutsche Übersetzung enthaltend.

Erste Abtheilung

die vier Evangelien.

$$
\begin{aligned}
& \text { G ö t t i n g e n, } \\
& \text { bei Vandenhoeck und } R \text { uprecht. } \\
& 1829 \text {. }
\end{aligned}
$$

Abbildung 1. Titelblatt ( $p$. II) des ersten Bandes des Gesamtwerks, der Textausgabe mit Übersetzung von 1829 mit der Ankündigung eines „kritischen und exegetischen Kommentars". 
exegetischen Kommentar“. Gedruckt war das Werk beim Göttinger Universitätsverlag Vandenhoeck und Ruprecht.

Es handelte sich also um ein Gesamtunternehmen mit zwei Teilen: (a) dem Text- und Übersetzungsteil und (b) dem Kommentarteil. Der erste Band der Kommentierung, der die Auslegung der drei ersten Evangelien umfasste, erschien dann auch 1832. ${ }^{2}$ Er hat eine besonders umfangreiche Titelei: ${ }^{3}$ Auf p. II findet sich wieder der Titel des Gesamtunternehmens: „Das Neue Testament griechisch usw.“, aber jetzt heißt es in der unteren Hälfte: „Zweiter Theil den Kommentar enthaltend“; rechts davon, auf p. III folgt der Obertitel der Kommentarreihe: „Kritisch exegetischer Kommentar über das Neue Testament“, und erst wenn man weiterblättert, findet man auf $\mathrm{p}$. v den Titel des betreffenden Bandes selbst (vgl. Abb. 2), ${ }^{4}$ in den ersten Jahrzehnten interessanterweise als „Handbuch“ bezeichnet. $^{5}$

Auffallend ist, dass im Titel des Gesamtwerks (Abb. 1) von einem „kritischen und (!) exegetischen Kommentar" die Rede ist, der direkt gegenüber stehende Reihentitel für die Kommentarreihe (p. III, ohne Abb.) dagegen von einem „Kritisch exegetischen Kommentar“ spricht und der einzelne Band ebenfalls „Kritisch exegetisches Handbuch“ (vgl. Abb. 2) heißt, also jeweils ohne „und“, allerdings auch ohne Bindestrich. Das weist auf einen unterschiedlichen Gebrauch von „kritisch“ hin. „Kritisch“ meint bei Meyer immer „textkritisch“. So beginnen die Kommentierungen jedes Kapitels immer mit einem Abschnitt zur Textkritik, erst dann folgen die exegetischen Ausführungen. Zum Doppelbegriff „kritisch exegetisch“ ist die Wortfügung erst später geworden, den Bindestrich, der das dann endgültig zum Ausdruck bringt, gibt es erst seit 1893.

des 20. Jahrhunderts eingeführt und erscheint erstmalig im KEK bei J. Weiß, Der erste Korintherbrief (KEK v; Göttingen: Vandenhoeck \& Ruprecht, 1910 ${ }^{9}$ ), xlviii + 388 Seiten.

2 H. A. W. Meyer, Kritisch exegetisches Handbuch über die Evangelien des Matthäus, Markus und Lukas (KEK I; Göttingen: Vandenhoeck und Ruprecht, 1832), xv + 419 Seiten; zur Titelei vgl. die Wiedergabe bei D.-A. Koch, „Abbildungen“, Der „Kritisch-exegetische Kommentar“ in seiner Geschichte (s. Anm. 1) 531-56, hier 536-8.

3 Titelei und Vorwort wurden lange Zeit mit römischen Ziffern gezählt, wobei dann die Kommentierung neu mit arabischer Seitenzählung einsetzte. Ab 1928 wurden die römischen Ziffern durch arabische Ziffern ersetzt und mit einem * als Zusatz gezählt (also: $4^{*}$ ); erst ab 1966 gibt es eine einheitliche Zählung für den jeweiligen Band insgesamt.

4 H. A. W. Meyer, Handbuch über Matthäus, Markus und Lukas (s. Anm. 2), p. v; Druckvorlage: Bibliothek des Landeskirchenamts der Ev. Kirche von Westfalen, Bielefeld; die Fotografie wurde von der Bibliothek zur Verfügung gestellt.

5 Dieser Titel wurde bis 1888 beibehalten. Seit 1890 trägt der einzelne Kommentar den Titel der kommentierten Schrift. Für weitere Informationen zur Entwicklung der Reihen- und Bandtitel vgl. D.-A. Koch, „Chronologie der Ausgaben des von H.A.W. Meyer begründeten Kritisch-exegetischen Kommentars“, Der „Kritisch-exegetische Kommentar“ in seiner Geschichte (s. Anm. 1) 471-504, hier 473-5. 


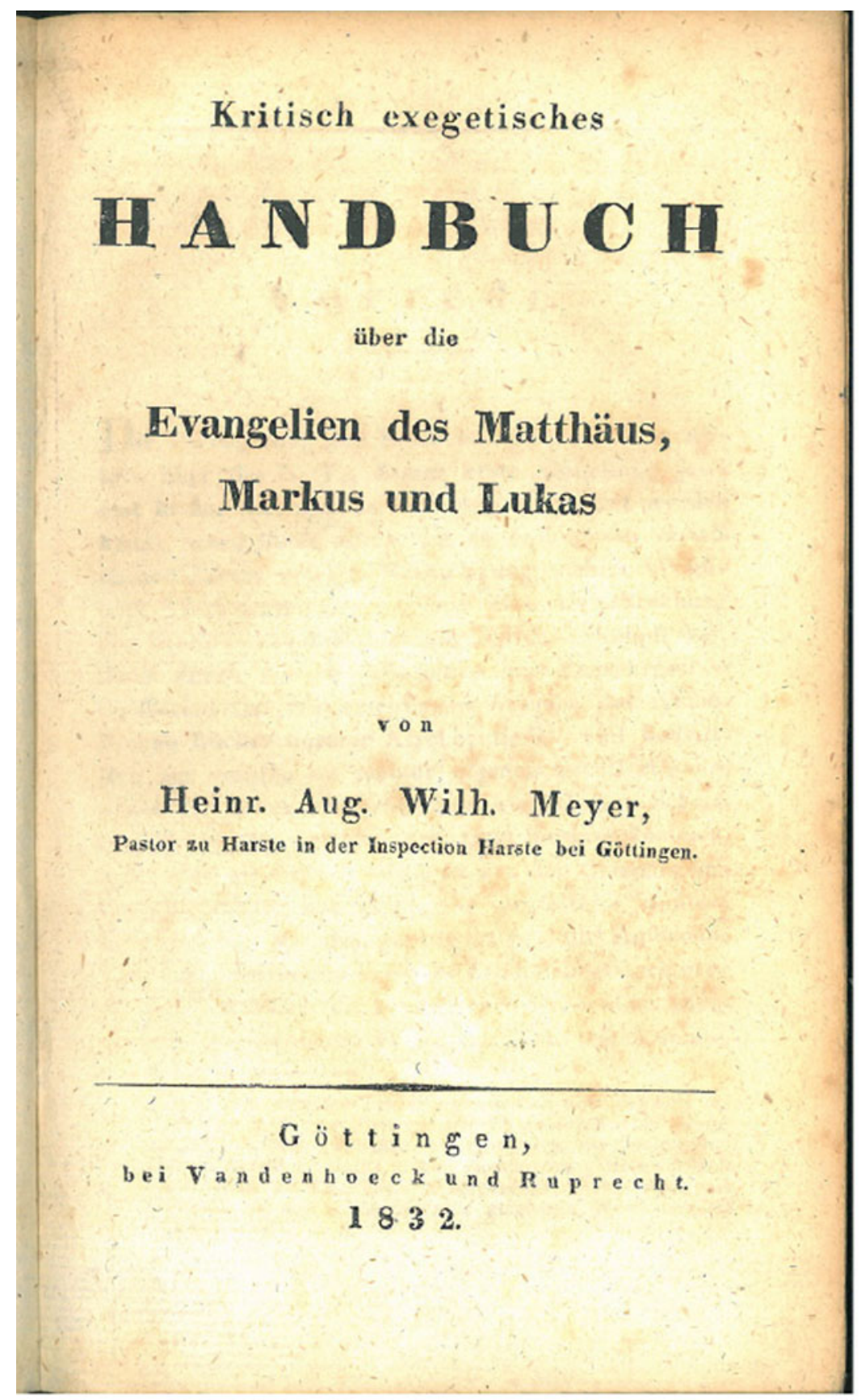

Abbildung 2. Titel des ersten Kommentarbandes: Kritisch exegetisches Handbuch über die Evangelien des Matthäus, Markus und Lukas von 1832 (p. v). 
Der Verfasser, Heinrich August Wilhelm Meyer, war 1832 inzwischen Dorfpfarrer im Königreich Hannover, ${ }^{6}$ und zwar in der Nähe von Göttingen; er hatte ursprünglich nur einen zweibändigen Kommentar zum ganzen Neuen Testament geplant, parallel zur ebenfalls zweibändigen Text- und Übersetzungsausgabe. Schon mit dem ersten Kommentarband von 1832, der die drei ersten Evangelien umfasste, jedoch nicht das Johannesevangelium, war dieser Plan faktisch schon aufgegeben worden. Als 1869 die Kommentierung des gesamten Neuen Testamentes abgeschlossen war, war das Kommentarwerk auf 16 Bände angewachsen, damals „Abtheilungen“ genannt, eigentlich sogar auf 17, denn bereits in der zweiten Auflage 1844 musste Meyer den besonders umfangreichen Band $\mathrm{zu}$ den drei ersten Evangelien in zwei Teilbände unterteilen. $^{7}$

Der Stahlstich (Abb. 3) ${ }^{8}$ zeigt Meyer im Alter von 45 bis 50 Jahren, also bereits auf der Höhe seines Erfolgs. Aus den frühen Jahren, in denen er das Projekt auf den Weg gebracht hat, gibt es noch keine derartig repräsentative Darstellung.

Das 1829 begonnene Projekt war, wie erwähnt, 1869, also nach 40 Jahren vollendet. In der Zwischenzeit bis 1869 hatte sich allerdings viel getan: Die ersten Bände waren bereits in der dritten bzw. vierten, ja z.T. in der fünfte überarbeiteten Auflage erschienen. ${ }^{9}$ Gleichzeitig wurden die letzten sieben Bände ab 1850 von anderen Autoren verfasst, da durch die fortlaufende Überarbeitung der bereits vorhandenen Bände das Werk Dimensionen angenommen hatte, die ein einzelner Autor nicht mehr bewältigen konnte. ${ }^{10}$

Woran lag der Erfolg dieser Kommentarserie, die es ja bis heute gibt? Meyer, der nie eine akademische Stellung an einer Universität oder einer höheren Lehranstalt innehatte, sozusagen ein Nobody in der Fachwelt, ${ }^{11}$ hatte eine

6 Zur Vita von H. A. W. Meyer vgl. K. Hammann, „Heinrich August Wilhelm Meyer (1800-1873) ein Lebensbild“, Der „Kritisch-exegetische Kommentar“ in seiner Geschichte (s. Anm. 1), 13-38.

7 H. A. W. Meyer, Kritisch exegetisches Handbuch über das Evangelium des Matthäus (KEK I/1; Göttingen: Vandenhoeck und Ruprecht, zweite verbesserte und vermehrte Auflage, 1844), XVI + 491 Seiten; zwei Jahre später folgte die Bearbeitung der beiden anderen synoptischen Evangelien: H. A. W. Meyer, Kritisch exegetisches Handbuch über die Evangelien des Markus und Lukas (KEK I/2: Göttingen: Vandenhoeck und Ruprecht, zweite verbesserte und vermehrte Auflage, 1846), 493 Seiten.

8 Vorlage: Privatbesitz des Verfassers.

9 Übersicht über sämtliche Bände des KEK von 1829 bzw. 1832 bis 2016: Koch, „Chronologie“ (s. Anm. 5), 471-504.

10 Es handelte sich um Gottlieb Lünemann (1850: 1/2 Thess; 1855: Hebr); Johannes Huther (1850: 1/2Tim, Tit; 1852: 1/2 Petr, Jud; 1855: 1-3 Joh; 1858: Jak) und Friedrich Düsterdieck (1859: Offb). Zu diesen Autoren vgl. die Biogramme in: „Die Autoren und Herausgeber des kritisch-exegetischen Kommentars“, Der „Kritisch-exegetische Kommentar" in seiner Geschichte (s. Anm. 1), 505-30, hier 509, 512, 516.

11 Daher war es eine mutige und - wie sich herausstellen sollte - für den Verlag zugleich wegweisende Entscheidung, dass der damalige Verleger Carl August Adolph Ruprecht das ambitionierte Projekt eines unbekannten thüringischen Dorfpfarrers in sein Verlagsprogramm 


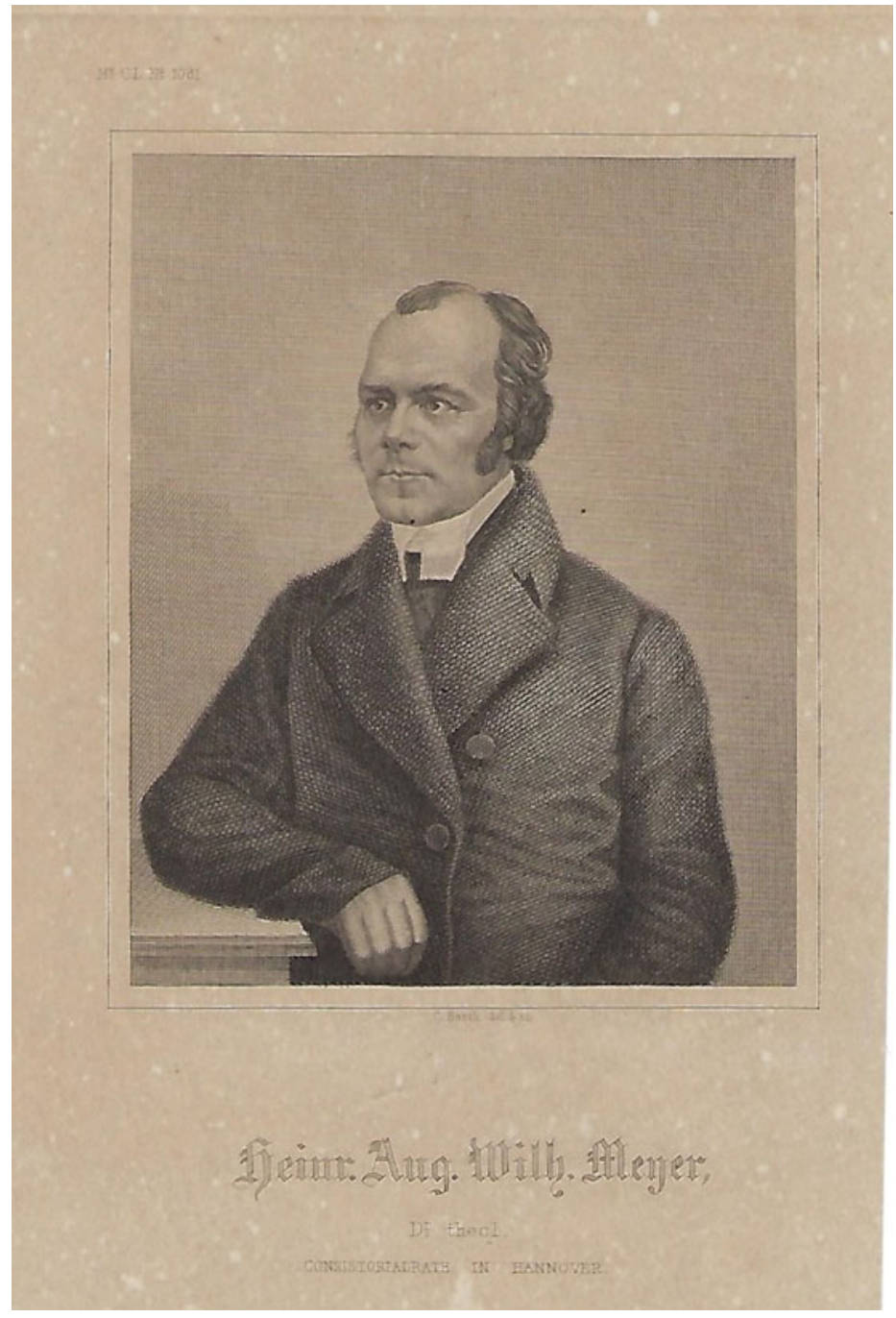

Abbildung 3. H. A. W. Meyer im Alter von 45 bis 50 Jahren.

Zielsetzung, mit der er auf Distanz zu den damals dominanten Strömungen in der protestantischen Theologie ging. Er forderte das „unbefangene, historisch grammatische von keiner Zeitphilosophie beherrschte, Studium des Neuen

aufnahm. Zu den Hintergründen vgl. Hammann, „Heinrich August Wilhelm Meyer (18001873) - ein Lebensbild“ (s. Anm. 6), 21f. 
Testaments“. ${ }^{12}$ Er wehrte sich sowohl gegen eine philosophisch-theologische Überfrachtung des Textes, andererseits gegen eine konfessionalistische Auslegung, deren vordringliches Interesse es war, die Lehrsätze der lutherischen Bekenntnisschriften im Neuen Testament wiederzufinden. So schreibt er ebenfalls 1829 im Vorwort zur Textausgabe über seine künftige Auslegung:

Denn den Sinn, wie ihn der S c h r i f t s t e 11 e $\mathrm{r}^{13}$ bei seinen Worten gedacht hat, ganz unpartheiisch, historisch grammatisch zu eruiren, - das ist die Pflicht des Exegeten; in welchem Verhältnis aber dieser eruirte Sinn zu den Lehren der Philosophie stehe, wie er mit den Dogmen der Kirche, oder mit den Ansichten ihrer Theologen stimme, wie ihn der Dogmatiker für seine Wissenschaft zu verarbeiten habe, - das geht den Exegeten, als Solchen, nichts an. ${ }^{14}$

Hier kämpft ein Verfasser für die Eigenständigkeit und das Eigenrecht der Exegese innerhalb von Theologie und Philosophie, und genau in diesem Sinne ist auch das Projekt eines exegetischen Kommentars zu verstehen.

Gegenüber der damals weit verbreiteten Tendenz, die neutestamentlichen Texte mithilfe der Philosophie des Deutschen Idealismus zu interpretieren, kann Meyer seine Kritik auch durchaus witzig formulieren. Im Vorwort zur Synoptikerauslegung von 1832 schreibt er: Er werde sich nie mit einer Exegese anfreunden, „welche den Sinn der ntl. Aussprüche philosophisch zurichtet, als ob Jesus und Paulus zu den Füssen Kant's oder Schellings gesessen hätten “15.

Damit hatte Meyer einen eigenen Weg zwischen den damaligen großen Lagern der Theologie gefunden, einen Mittelweg, durch den seine Kommentare nicht auf eine bestimmte inhaltliche Linie festgelegt waren, sondern deren Markenzeichen die philologische Methode war. Auch gegenüber den Konstruktionen der Tübinger Tendenzkritik ${ }^{16}$ blieb er skeptisch und hat ebenso gegen pietistische Verbiegungen Position bezogen. Diese Unabhängigkeit im Zugang zu den Texten führte offenbar zu der breiten Akzeptanz der Kommentarreihe und sicherte so auch deren verlegerischen Erfolg. ${ }^{17}$

Bei den einzelnen exegetischen Streitfragen war er dementsprechend sehr zurückhaltend. Dabei ist nicht so sehr das Festhalten an der Echtheit des

12 Vorwort zur Textausgabe H. A. W. Meyer, Das Neue Testament Griechisch, Bd. I (Göttingen: Vandenhoeck und Ruprecht, 1829; s. Abb. 1) x.

13 Sperrung im Original.

14 Meyer, Das Neue Testament Griechisch (s. Abb. 1), I.XxxI.

15 Meyer, Kritisch exegetisches Handbuch über die Evangelien des Matthäus, Markus und Lukas, XI.

16 Zur „Tübinger Schule“ vgl. U. Köpf, „Tübinger Schulen“, TRE 34 (2002) 165-71, hier 167-70. $17 \mathrm{Zu}$ dem von Meyer begründeten Kommentarwerk aus der Sicht des Verlages vgl. den Beitrag des Altverlegers A. Ruprecht, „Der Kritisch-exegetische Kommentar als Verlagsprojekt“, Der "Kritisch-exegetische Kommentar" in seiner Geschichte (s. Anm. 1), 62-9. 
Kolosser- und Epheserbriefs auffällig, ${ }^{18}$ wichtig ist aber, dass er diese Fragen von Auflage zu Auflage immer wieder durchdenkt und Früheres z.T. deutlich revidiert. Besonders bemerkenswert ist, dass er 1853, bei der dritten Auflage des MtKommentars, ${ }^{19}$ dazu übergeht, die Mk-Priorität vorauszusetzen. ${ }^{20}$ Hier, wie auch in anderen Kommentaren, kann man sehen, wie Meyer nicht nur exegetische Meinungen referierte, sondern auch bestrebt war, sie ernsthaft $\mathrm{zu}$ bedenken.

Damit sind wir bereits bei dem zweiten Grund für den Erfolg der Kommentarreihe, der ständigen Aktualisierung der Kommentare. Schon 1844, nach zwölf Jahren, Meyer war mit den neu zu bearbeitenden Texten bis zum Epheserbrief $^{21}$ vorgestoßen, da erschien bereits die zweite Auflage des MtKommentars, jetzt als selbständiger Band, dem dann 1846 die zweite Auflage des Kommentars zu Markus und Lukas folgte. Es gab dann nur noch eine Neuedition durch ihn selbst, die sog. „9. Abtheilung“, die die Kommentare über den Philipper-, Kolosser- und Philemonbrief umfasste, ansonsten nur noch Aktualisierungen. Hatte Meyer in den ersten zwölf Jahren acht Neubearbeitungen herausgebracht, so folgten in den anschließenden 30 Jahren, also von 1844 bis zu seinem Tod 1873, insgesamt 39 Bände, eine Neubearbeitung (Phil/Kol/Phlm) und 38 Neuauflagen, also mehr als ein Band pro Jahr. Dabei wurden die Abstände zwischen den Neuauflagen pro kommentierter Schrift immer kürzer, vielfach nur fünf oder sechs Jahre, was sicher auch mit der sich steigernden Nachfrage zu tun hatte. ${ }^{22}$ Das sind natürlich Zahlen, die man heute nie erreichen kann. Auch wenn das Arbeitspensum von Meyer sicher enorm gewesen sein muss - die Menge der zu verarbeitenden Literatur und deren regelmäßige Vermehrung hat heute ganz andere Dimensionen erreicht.

Als Meyer, zum Schluss längst nicht mehr Dorfpfarrer, sondern Superintendent und Konsistorialrat in Hannover und Ehrendoktor der

18 Dazu vgl. in dem Band Der „Kritisch-exegetische Kommentar“ in seiner Geschichte (s. Anm. 1) die Beiträge im Mittelteil, die die Geschichte der Kommentierung der einzelnen neutestamentlichen Schrift jeweils von der ersten bis zur derzeit letzten Auflage behandeln, zum Kol: P. Müller, „Der Kolosserbrief“, 309-23, hier 313f; zum Eph: A. Lindemann, „Der Epheserbrief“, 268-86, hier 273f.

19 H. A. W. Meyer, Kritisch exegetisches Handbuch über das Evangelium des Matthäus (KEK I/1; Göttingen: Vandenhoeck und Ruprecht, dritte verbesserte und vermehrte Auflage, 1853), XII + 499 Seiten.

20 Vgl. W. Kraus, „Das Matthäusevangelium“, Der „Kritisch-exegetische Kommentar“ in seiner Geschichte (s. Anm. 1), 85-105, hier 94f und C. Breytenbach, „Das Markusevangelium“, ebd. 106-23, hier 107f.

21 H. A. W. Meyer, Kritisch exegetisches Handbuch über den Brief an die Epheser (KEK VIII; Göttingen: Vandenhoeck und Ruprecht 1843), XIV + 269 Seiten.

22 Reine Nachdrucke sind dagegen selten; ein solcher Nachdruck erfolgte 1865, als von der vierte Auflage der Kommentare zum 1. und 2. Korintherbrief (1861 bzw. 1862) eine „Zweite Ausgabe" erschien. 
Theologischen Fakultät Göttingen, im Jahre 1873 starb, hatte er längst mit dem Verlag Vandenhoeck und Ruprecht die Fortführung des Kommentarwerks geregelt. Das war ein entscheidender Schritt für die Fortexistenz der Reihe über den Tod ihres Begründers hinaus. Schon 1850, nach einer schweren Krankheit, hatte Meyer für die letzten verbleibenden sieben Bände weitere Autoren herangezogen. Damit hatte er bereits zu Lebzeiten den Kommentar ein Stück weit von seiner Person abgelöst. Das geschah jetzt endgültig. Die Verantwortung für die Fortführung lag von nun an bei dem jeweiligen Verleger, der die neuen Bearbeiter gewinnen und bestimmen musste. ${ }^{23}$ Erst ab 1971 gibt es einen eigenen Herausgeber für den KEK.

Von 1832, als Meyer den ersten Kommentarband veröffentliche, bis zu seinem Tod 1873 hat sich der Kommentar bereits erheblich gewandelt. Dominierte zunächst im exegetischen Teil der sog. glossatorische Methode, also das Verfahren, am Text entlang philologische und exegetische Beobachtungen aus der Auslegungsgeschichte zu sammeln und dazu Stellung zu beziehen, so ging Meyer selbst im Laufe der Zeit dazu über, diese glossatorische Kommentierung durch thematische Erörterungen zu ergänzen.

Auf dieser letzten Stufe der Entwicklung von Meyer's Kommentar erfolgte eine Übersetzung ins Englische, die beim Verlag T. \& T. Clark in Edinburgh ab 1873 erschien. ${ }^{24}$ Hauptherausgeber war William P. Dickson, ${ }^{25}$ in Zusammenarbeit mit Frederick Crombie $^{26}$ und William Stewart. ${ }^{27}$ Die insgesamt 20 Bände

23 Dabei waren bis 2016 insgesamt - einschließlich von H. A. W. Meyer selbst - insgesamt 40 Autoren an dem Kommentarwerk beteiligt; vgl. dazu auch den Beitrag von E.-M. Becker, „Die Person des Kommentators als wissenschaftliche persona. Zur Beschreibung eines neuen Arbeitsfeldes“, Der „Kritisch-exegetische Kommentar“ in seiner Geschichte (s. Anm. 1), $70-82$.

24 Der Reihentitel des ersten erschienenen Bandes auf lautet (p. I): „Critical and Exegetical Commentary on the New Testament by Heinrich August Wilhelm Meyer. From the German, with the Sanction of the Author. The Translation Revised and Edited by William P. Dickson. Part Iv. The Epistle to the Romans. Vol. I. Edinburgh, T. \& T. Clark. 1873“ (s. Abb. 4). Der Titel des jeweiligen Bandes (auf p. III) folgt eng dem Vorbild von H. A. W. Meyer: „Critical and Exegetical Handbook to the Epistle to the Romans by Heinrich August Wilhelm Meyer. Translated from the Fifth Edition of the German", gefolgt von der Angabe des Übersetzers.

- Die Abfolge der Veröffentlichung der einzelnen Bände hat offenbar nur technische Gründe.

25 William P. Dickson (1823-1901) war 1863-95 „Professor of Divinity” an der Universität Glasgow. Er veröffentlichte u.a. die Untersuchung: St. Paul's Use of the Terms Flesh and Spirit (Glasgow: J. Maclehose \& Sons, 1883). Hervorgetreten ist er außerdem durch eine englische Übersetzung von Theodor Mommsens Römische Geschichte: The History of Rome (London: R. Bentley, 1864), erschienen in fünf Bänden.

26 Frederik Crombie (1827-89) war 1868-89 „Professor of Divinity“ an der Universität St Andrews.

27 William Stewart (1835-1919) war 1873-1910 „Professor of Biblical Criticism“ an der Universität Glasgow. 
umfassende Ausgabe begann mit dem Kommentar zum Römerbrief, dessen erster Band 1873 erschien (Abb. 4). ${ }^{28}$

Er enthielt neben einer ausführlichen Einleitung, in der der Herausgeber Meyer's Kommentarwerk würdigt (p. v- XVII), auch ein von Meyer selbst verfasstes und auf „March 1873“ datiertes Vorwort: „Preface Specially Written by the Author for the English Edition“ (p. xxxI - xxxv). Während der Drucklegung war Meyer verstorben (†21.6.1873), so dass der Herausgeber sein Vorwort um eine persönliche Würdigung ergänzte und einen kurzen biographischen Abriss, verfasst von Meyer's Sohn Gustav Meyer, anfügte. Übersetzt wurden sämtliche von Meyer selbst verfassten Kommentarbände, und zwar jeweils in der letzten von ihm selbst besorgten Auflage. ${ }^{29}$ Die von Gottlieb Lünemann und Johannes Eduard Huther verfassten Kommentare (1/2 Thess; Past; 1/2 Petr, Jud, Hebr, 1 - 3 Joh) wurden ebenfalls übersetzt, aber nicht mehr unter der Verantwortung von W. P. Dickson. ${ }^{30}$ Die Übersetzung des Kommentars zur Johannesoffenbarung durch Friedrich Düsterdieck unterblieb aus Mangel an Interesse ${ }^{31}$ seitens der Subskribenten.

Unmittelbar danach, von 1873 bis 1887 erschien in New York bei Verlag Funk \& Wagnalls eine weitere, allerdings unautorisierte englische Ausgabe des KEK. Sie war eine Wiedergabe der bei T. \& T. Clark erschienenen Übersetzung, ergänzt um

28 Vorlage: Privatbesitz des Verfassers.

29 In zwei Fällen war bereits eine nach dem Tode Meyers erfolgte Neubearbeitung der deutschen Kommentierung erschienen. Es handelt sich um: B. Weiß, Kritisch exegetisches Handbuch über die Evangelien des Markus und Lukas (KEK I/2; Göttingen: Vandenhoeck und Ruprecht, $1878^{6}$ ) und W. Schmidt, Kritisch exegetisches Handbuch über den Brief an die Epheser (KEK vII; Göttingen: Vandenhoeck und Ruprecht, $1878^{5}$ ). Auch in diesen Fällen wurde die letzte Bearbeitung durch H. A. W. Meyer selbst der Übersetzung zugrunde gelegt; zur Begründung schreibt W. P. Dickson, „Prefatory Note by the Editor“, in H. A. W. Meyer, Critical and Exegetical Handbook to the Gospels of Mark and Luke (hg. W. P. Dickson; Edinburgh: T. \& T. Clark, 1880) v-VII, hier vI in Blick auf die Neubearbeitung durch B. Weiß: „the work is no longer such as Meyer left it; it is to a considerable extent a new book by another author, and from a standpoint in various respects different". Vgl. die analoge Begründung in W. P. Dickson, „Prefatory Note by the Editor“, in H. A. W. Meyer, Critical and Exegetical Handbook to the Epistle to the Ephesians and the Epistle to Philemon (Edinburgh: T. \& T. Clark, 1880), v-VIII, hier v-VI.

30 In diesen Bänden fehlt eine Herausgeberangabe überhaupt. Im letzten von ihm selbst herausgegebenen Band schreibt Dickson, dass er sich nur verpflichtet fühlte, die englische Übersetzung der von Meyer selbst verfassten Bände als Herausgeber zu verantworten; so W. P. Dickson, „Prefatory Note by the Editor“, Handbook to the Epistle to the Ephesians (s. Anm. 29), p. viII.

31 Letzter Band war der Kommentar zum Hebräerbrief von 1882: G. Lünemann, Critical and Exegetical Handbook to the Epistle to the Hebrews (Edinburgh: T. \& T. Clark, 1882): auf p. II findet sich folgende "Notice to the Subscribers. - This Issue completes the Series of MEYER'S COMMENTARIES ON THE NEW TESTAMENT. In Twenty Volumes.“ Es folgt eine Übersicht über alle 20 Bände, an die sich die Nachbemerkung anschließt: „DÜSTERDIECK ON REVELATION will not be translated in the meantime, as the Publishers have received no encouragement from the Subscribers." 


\section{CRITICAL AND EXEGETICAL}

\section{O MMENTAR Y}

oN

\section{THE NEW TESTAMENT.}

BY

\section{HEINRICH AUGUST WILHELM MEYER, TH.D. \\ odercoNsistoriaLrath, HANNOVER. \\ from the German. \\ THE TRANSLATION REVISED AND EDITED, WITH THE SANCTION OF THE AUTHOR, BY}

WILLIAM P. DI CKSON, D.D.,

PROFESSOR OF DIVINITY IN THE UNIVERSITY OF GLASGOW.

PART IV.

THE EPISTLE TO THE ROMANS.

VOL. I.

E D I N B U GH:

T. AND T. CLARK, 38 GEORGE STREET. MDCCCLXXIII

SCOTTISH BAPTIST COLLEGE

Abbildung 4. Titel des ersten Bandes der englischen Übersetzung, herausgegeben von W. P. Dickson, Edinburgh 1873 (p. I). 
die Übersetzung des Kommentars zur Apokalypse von Friedrich Düsterdieck, wobei es keinen Reihenherausgeber gab, sondern sich acht Herausgeber die Editionsarbeit teilten. ${ }^{32}$ Diese elf Bände umfassende Ausgabe enthielt außer einem eigenen Vorwort des jeweiligen Herausgebers auch „Notes by the American Editor“, die nach jedem Kapitel anfügt wurden. In diesen „Notes“ führt der Herausgeber ergänzende und abweichende Positionen aus der neueren Literatur an, berücksichtigt dabei auch die inzwischen erschienenen Neubearbeitungen des „Meyer“ (z.B. durch Bernhard Weiß) und bezieht auch selbst - z.T. sehr dezidiert - Stellung. ${ }^{33}$

Nach dem Tode von Meyer 1873 lag, wie bereits erwähnt, die Fortführung des Kommentars allein in den Händen des zuständigen Verlegers, Carl Johann Friedrich Wilhelm Ruprecht, der den Verlag von 1861 bis 1897 führte. Es war offenbar der Göttinger Systematiker Albrecht Ritschl, ${ }^{34}$ der den Verleger auf den jungen aufstrebenden Neutestamentler Bernhard Weiß, damals noch in Kiel, hinwies. Bernhard Weiß war in der Tat die entscheidende Figur in dieser kritischen Phase. ${ }^{35}$

Er bearbeitete zwischen 1878 und 1902 insgesamt sieben Bände („Abteilungen“) des Kommentars, und zwar alle Evangelien, den Römerbrief, die Pastoralbriefe, den Hebräerbrief und die Johannes-Briefe, jeweils in mehreren Auflagen, insgesamt 22 Bände, $^{36}$ und dies neben zahlreichen anderen Publikationen.

Mit dem immensen Einsatz von Bernhard Weiß war einerseits das Überleben der Reihe gesichert, andererseits aber auch eine starke persönliche Fixierung gegeben. Bernhard Weiß schrieb Meyers Kommentare fort, gestaltete sie auch um, ohne völlig neue Bahnen zu betreten. Immerhin wurde neben Bernhard

32 Dies waren: George R. Crooks (Mt), Matthew B. Riddle (Mk und Lk), A. C. Kendrick (Joh), William Ormiston (Apg), Timothy Dwight (Röm; Phil; Kol; Phlm; Kath. Briefe), Talbot W. Chambers (1/2 Kor), Henry J. Jacobs (Gal; Eph; Offb), Maurice J. Evans (Past, Hebr).

33 Der Titel ist mit dem der Ausgabe von T. \& T. Clark identisch, wobei zusätzlich der amerikanische Herausgeber angegeben ist.

$34 \mathrm{Zu}$ Albrecht Ritschl vgl. D.-A. Koch, „Ritschl, Albrecht“, Der „Kritisch-exegetische Kommentar“ in seiner Geschichte (s. Anm. 1), 527f (mit weiterführender Literatur). Zur theologiegeschichtlichen Stellung Ritschls vgl. auch E. Lessing, Geschichte der deutschsprachigen evangelischen Theologie von Albrecht Ritsch bis zur Gegenwart, Bd. I: 1870-1918 (Göttingen: Vandenhoeck \& Ruprecht, 2000) 33-43.

35 Zur Geschichte des Kommentarwerks insgesamt vgl. F. W. Horn, „Von ,Meyers Kommentar zum ,Kritisch-exegetischen Kommentar` (KEK): Beobachtungen zur Geschichte eines neutestamentlichen Kommentarwerks“, Der „Kritisch-exegetische Kommentar“ in seiner Geschichte (s. Anm. 1), 39-61; zu Bernhard Weiß vgl. auch W. Kraus, „Weiß, Karl Philipp Bernhard“, ebd. $521 f$ (mit Aufstellung sämtlicher von ihm bearbeiteter Kommentare).

36 Dabei wurden die Auflagenziffern grundsätzlich fortgeführt, was spätestens bei dem dritten Bearbeiter einer Schrift zu unrealistisch hohen Auflagenziffern führte. Dem ist in jüngerer Zeit dadurch Rechnung getragen, dass die Neubearbeitungen zusätzlich eine eigene Zählung erhielten (also: „1. Auflage dieser Neubearbeitung“ usw.). 
Weiß der damals in Marburg lehrende Georg Heinrici mit der Bearbeitung der Korintherkommentare betreut, ${ }^{37}$ der sich schrittweise von den Meyerschen Vorgaben löste und neues religionsgeschichtliches Material in die Kommentierung mit einbezog.

Nicht zufällig gründete Heinrici 1915 das Forschungsprojekt des „Corpus Hellenisticum“; ${ }^{38}$ aus diesem Bereich stammen auch Heinricis Schüler Eduard von Dobschütz, Martin Dibelius und Hans Windisch, die dann alle Kommentatoren des KEK wurden. Allerdings geschah dies mit einiger zeitlichen Verzögerung: Dobschütz' Kommentar zu den Thessalonicherbriefen erschien 1909, ${ }^{39}$ die Kommentare von Dibelius und Windisch erst nach dem Ersten Weltkrieg, 1921 Dibelius zu Jakobus, ${ }^{40}$ und 1924 Windisch zum 2. Korintherbrief. ${ }^{41}$ Auch für die Vertreter der religionsgeschichtlichen Schule im engeren Sinne galt dies genauso: Der einzige hier schon früh zu nennende Autor ist Wilhelm Bousset.

Bousset, übrigens mit dem Hause Ruprecht persönlich verbunden, veröffentlichte 1896 den Kommentar zur Offenbarung des Johannes, ${ }^{42}$ was nun

37 G. Heinrici, Kritisch exegetisches Handbuch über den ersten Brief an die Korinther (KEK v; Göttingen: Vandenhoeck und Ruprecht, $\left.1881^{6}\right), x+479$ Seiten; die Folgeauflagen stammen von 1888 (siebte Auflage), 1896 (achte Auflage); und: Kritisch exegetisches Handbuch über den zweiten Brief an die Korinther (KEK v; Göttingen: Vandenhoeck und Ruprecht, $1883^{6}$ ), VIII + 406 Seiten; die Folgeauflagen stammen von 1890 (siebte Auflage) und 1900 (achte Auflage); zu G. Heinrici vgl. D.-A. Koch, „Heinrici, (Carl Friedrich) Georg“, Der „Kritisch-exegetische Kommentar“ in seiner Geschichte (s. Anm. 1), $511 \mathrm{f}$.

38 Dazu vgl. P. W. van der Horst, „Corpus Hellenisticum Novi Testamenti”, Anchor Bible Dictionary, vol. I (1992) 1157-61.

39 E. v. Dobschütz, Die Thessalonicher-Briefe (KEK x; Göttingen: Vandenhoeck und Ruprecht, $\left.1909^{7}\right), x+320$ Seiten; 1974 erfolgte ein Nachdruck (mit einem Vorwort von F. Hahn); zu E. v. Dobschütz vgl. U. Mell, „Dobschütz, Ernst (Adolf Alfred Oskar Adalbert) von“, Der „Kritisch-exegetische Kommentar" in seiner Geschichte (s. Anm. 1), 509.

40 M. Dibelius, Der Brief des Jakobus (KEK xv; Göttingen: Vandenhoeck \& Ruprecht, 1921 ${ }^{7}$ ), VI + 240 Seiten; die achte Auflage von 1956 ist ein Nachdruck, zu dem gleichzeitig ein Ergänzungsheft erschien (herausgegeben von H. Greeven), das Berichtigungen und Ergänzung aus dem Handexemplar von M. Dibelius enthielt und Literaturnachträge von H. Greeven aus der neueren Literatur; die neunte Auflage (1957) ist ein Nachdruck, die zehnte Auflage von 1959 ebenso (wobei das Ergänzungsheft erweitert ist). In der 11. Auflage von 1964 ist das Ergänzungsheft in die neugesetzte Auflage eingearbeitet; hiervon ist die 12. Auflage von 1984 ein Nachdruck (mit weiteren Anhängen); zu M. Dibelius vgl. O. Wischmeyer, „Dibelius, Martin (Franz)“, Der „Kritisch-exegetische Kommentar“ in seiner Geschichte (s. Anm. 1), 508f, zur forschungsgeschichtlichen Bedeutung des Jak-Kommentars von Dibelius vgl. O. Wischmeyer, „Der Jakobusbrief“, ebd., 436-53, hier 443-5.

41 H. Windisch, Der zweite Korintherbrief (KEK vi; Göttingen: Vandenhoeck \& Ruprecht, 1924 ${ }^{9}$ ), VII + 436 Seiten; 1970 erfolgte ein Nachdruck (mit einem Vorwort von G. Strecker); zu H. Windisch vgl. D.-A. Koch, „Windisch Hans“, Der „Kritisch-exegetische Kommentar“ in seiner Geschichte (s. Anm. 1), 524.

42 W. Bousset, Die Offenbarung Johannis (KEK xv; Göttingen: Vandenhoeck und Ruprecht, $\left.1896^{5}\right)$, VI +527 Seiten; die erheblich umgearbeitete und bis heute maßgebliche sechste 
auch wieder bezeichnend ist, denn das war das Buch, das in der bisherigen Exegese - und auch im „Kritisch-exegetischen Kommentar“ - keinen besonders guten Stand hatte ${ }^{43}$ und mit dem jedoch Hermann Gunkel 1894 in seinem Buch „Schöpfung und Chaos“44 seine neue, die religionsgeschichtliche Methode programmatisch exemplifizierte und damit die religionsgeschichtliche Schule im eigentlichen Sinne begründet hat. ${ }^{45}$ Erst 1910 erschien mit Johannes Weiß der nächste Kommentar aus der religionsgeschichtlichen Schule, nämlich zum 1. Korintherbrief. ${ }^{46}$

Der alte Tanker „Meyerscher Kommentar“ war also nicht so leicht umzusteuern. Die neue Verlegergeneration, der religionsgeschichtlichen Schule und ihren Vertretern auch persönlich durchaus verbunden, erschuf daher im Verlag ein eigenes religionsgeschichtliches Kommentarwerk ${ }^{47}$ neben dem KEK, die von Johannes Weiß in zwei Bänden herausgegebenen: „Schriften des Neuen Testaments“. ${ }^{48} \mathrm{Im}$ „Meyer“ ging es, wie gesagt, langsamer voran. Aber: es ging

Auflage erschien 1906 (Iv + 468 Seiten); sie wurde 1966 nachgedruckt; zu W. Bousset vgl. Th. Witulski, „Bousset, (Johann Franz) Wilhelm“, Der „Kritisch-exegetische Kommentar“ in seiner Geschichte (s. Anm. 1), 507.

43 Vor Bousset gab es im KEK lediglich einen Kommentator der Apk, Friedrich Düsterdieck (erste Auflage 1859; Folgeauflagen: 1865, 1877, 1887). Düsterdieck wendet sich zwar m.R. gegen die allegorische Auslegung der Apk, behandelt sie allerdings weiterhin als inspiriertes Buch, das jedoch mit den (s.E.) originär apostolischen Schriften nicht gleichrangig sei. Er betreibt damit eine „innerkanonisch-kritische Lektüre [der Apk] ..., ohne jedoch deren historisch-individuelle Dimension wirklich erfassen zu wollen“ (Th. Witulski, „Die Offenbarung des Johannes“, Der „Kritisch-exegetische Kommentar“ in seiner Geschichte (s. Anm. 1), 454-67, hier 455).

$44 \mathrm{H}$. Gunkel, Schöpfung und Chaos in Urzeit und Endzeit. Eine religionsgeschichtliche Untersuchung über Gen 1 und Ap Joh 12 (Göttingen: Vandenhoeck und Ruprecht, 1895).

$45 \mathrm{Zu}$ Hermann Gunkel vgl. K. Hammann, Hermann Gunkel. Eine Biographie (Tübingen: Mohr Siebeck, 2014), hier 58-72 zur forschungsgeschichtlichen Bedeutung von „Schöpfung und Chaos“. Zur „religionsgeschichtlichen Schule“ vgl. G. Lüdemann/A. Özen, „Religionsgeschichtliche Schule“, TRE 28 (1997), 618- 24.

46 J. Weiß, Der erste Korintherbrief (KEK v; Göttingen: Vandenhoeck \& Ruprecht, $1910^{9}$ ), XLVIII + 388 Seiten); Nachdrucke erfolgten 1925 (als zehnte Auflage), und 1970 und 1977 (jeweils ohne Auflagenzählung); zu Johannes Weiß vgl. D.-A. Koch, „Weiß, Johannes“, Der „Kritisch-exegetische Kommentar" in seiner Geschichte (s. Anm. 1), 523.

47 Zur Entstehung der „Schriften des Neuen Testaments“ vgl. W. Ruprecht, Väter und Söhne. Zwei Jahrhunderte Buchhändler in einer deutschen Universitätsstadt (Göttingen: Vandenhoeck \& Ruprecht, 1935) 222f.

48 J. Weiß (Hg.), Die Schriften des Neuen Testaments neu übersetzt und für die Gegenwart erklärt (Göttingen: Vandenhoeck \& Ruprecht, Bd. I: 1906, Bd. II: 1907); eine zweite Auflage erschien 1907/8; die dritte Auflage, herausgegeben von W. Bousset und W. Heitmüller, erschien in vier Bänden 1917/18; deren erster Band (die drei ersten Evangelien) erlebte noch 1929 eine vierte Auflage. 
immerhin voran, während ja die „Schriften des Neuen Testaments“ ohne Nachfolge blieben. ${ }^{49}$

Für die Zeit nach dem Ersten Weltkrieg ist beides festzustellen, die Fortführung bisheriger Ansätze, d.h. der philologischen und religionsgeschichtlichen Kommentierung im Horizont der liberalen Theologie, dazu gehören, wie schon erwähnt, Dibelius und Windisch. ${ }^{50}$ Daneben stehen theologische Neuaufbrüche, die sich auf ganz spezifische Weise im „Meyer“ widerspiegeln, nämlich im Nebeneinander von Ernst Lohmeyer ${ }^{51}$ und Rudolf Bultmann. ${ }^{52}$

Von E. Lohmeyer stammen im KEK zwei vollständige Kommentarbände und ein Sonderband: der 1930 erschienene Kommentar zum Philipper-, Kolosser- und Philemonbrief;; ${ }^{53}$ sodann 1937 der Kommentar zum Markusevangelium ${ }^{54}$ und 1956 erschienen postum als Sonderband Lohmeyers Vorarbeiten für den geplanten Mt-Kommentar. ${ }^{55}$ Von R. Bultmann erschienen im KEK (wie von E. Lohmeyer) zwei Kommentarbände und ein Sonderband: von 1937 bis 1941 der

49 Zum Ende des sog. „Göttinger Bibelwerks“ nach dem Ersten Weltkrieg vgl. Ruprecht, Väter und Söhne, 223f. Zum „Nachfolgeprojekt“, dem „Neuen Testament Deutsch“ (NTD), das dann sehr anders ausgerichtet war als die „Schriften des Urchristentums“, vgl. Ruprecht, a. a.O., 273f.

$50 \mathrm{Zu}$ M. Dibelius vgl. E. Lessing, Geschichte der deutschsprachigen evangelischen Theologie von Albrecht Ritschl bis zur Gegenwart, Bd. II: 1918-1945 (Göttingen: Vandenhoeck \& Ruprecht, 2004) 219-223, 253-5; für H. Windisch ist auf seine scharfe Distanzierung von Karl Barth im Vorwort zum Kommentar zum 2. Korintherbrief von 1924 (s. Anm. 41), hier p. vI, zu verweisen.

51 Zu E. Lohmeyer vgl. E.-M. Becker „Lohmeyer, Ernst“, Der „Kritisch-exegetische Kommentar“ in seiner Geschichte (s. Anm. 1), 515; zusätzlich jetzt: Ch. Böttrich (Hg.), Ernst Lohmeyer. Beiträge $z u$ Leben und Werk (Greifswalder Theologische Forschungen 28, Leipzig: Evangelische Verlagsanstalt, 2018).

52 Zu R. Bultmann, vgl. D.-A. Koch, „Bultmann, Rudolf“, Der „Kritisch-exegetische Kommentar“ in seiner Geschichte (s. Anm. 1), 507f; die maßgebliche neuere Darstellung ist die von K. Hammann, Rudolf Bultmann. Eine Biographie (Tübingen: Mohr Siebeck, 2012 ${ }^{3}$ ).

53 Die Briefe an die Philipper, an die Kolosser und an Philemon (KEK IX, Göttingen: Vandenhoeck \& Ruprecht, $1930^{8}$ ), $4^{*}+192$ Seiten (Phil), 201 Seiten (Kol, Phlm, Register); die Kommentierung des Philipperbriefs erschien als Separatdruck bereits 1928; 1953 erschien postum (herausgegeben von W. Schmauch) eine durchgesehene Auflage, die viermal nachgedruckt wurde (zwischen 1954 und 1974 als 10. bis 13. Auflage; Phil wurde separat 1974 in 14. Auflage gedruckt); das Beiheft von Werner Schmauch von 1964 enthielt Literaturnachträge und Beiträge aus der neueren Diskussion.

54 Das Evangelium des Markus (KEK I/2; Göttingen: Vandenhoeck \& Ruprecht, $1937^{10}$ ), $6^{*}+368$ Seiten; 1951 erschien postum eine 11. durchgesehene Auflage mit einem Ergänzungsheft von Gerhard Saß, das die größeren Korrekturen aus dem Handexemplar Lohmeyers enthielt; der Kommentar wurde sechsmal nachgedruckt (zwischen 1953 und 1967 als 12. bis zur 17 . Auflage).

55 Das Evangelium des Matthäus. Nachgelassene Ausarbeitungen und Entwürfe (hg. W. Schmauch; KEK Sonderband; Göttingen: Vandenhoeck \& Ruprecht, 1956), 12* 429 Seiten; davon erschienen drei Nachdrucke (zwischen 1958 und 1967 als zweite bis vierte Auflage). 
Kommentar zum Johannesevangelium, ${ }^{56}$ dem erst 1967 der Kommentar zu den Johannesbriefen folgte; ${ }^{57}$ als Sonderband erschien 1976 der Kommentar zum 2. Korintherbrief. ${ }^{58}$

Angesichts der nach dem Ersten Weltkrieg ausgebrochenen Krise der liberalen Theologie wollten beide Autoren nicht bei der historisch orientierten Texterklärung verharren, sondern durchstoßen zu einer Begegnung mit der in den Texten gemeinten Sache, ${ }^{59}$ wobei Bultmann, dessen Kommentar zum JohEv in Lieferungen ab 1937 erschien, dabei die literarischen und religionsgeschichtlichen Fragen nicht überspringen wollte, sondern zum Ausgangspunkt für eine bewusst theologische Exegese benutzte. Bei Lohmeyer wird dagegen „der philologisch-historische Stoff fast durchweg in die Anmerkungen verwiesen“, so im Vorwort der Gesamtausgabe der neunten Abteilung von 1929, die den Philipper-, Kolosser- und Philemonbrief umfasste. ${ }^{60}$ Im diesem Vorwort sagt Lohmeyer auch ausdrücklich, „daß die Kommentare nicht nur im einzelnen nachgeschlagen, sondern im ganzen gelesen werden möchten “. ${ }^{61}$ Den gleichen Anspruch erhebt auch Bultmann in einem Brief an den Verlag von 1941, als der Verlag ihm mitteilte, ${ }^{62}$ nach dem Erscheinen der letzten Lieferung des

56 Das Evangelium des Johannes (KEK II; Göttingen: Vandenhoeck \& Ruprecht, $1941^{10}$ ), $12^{*}+567$ Seiten; der Kommentar erschien in sieben Lieferungen; die erste Lieferung datiert von 1937, mit der letzten Lieferung 1941 war der Kommentar vollständig; eine durchgesehene 11. Auflage erschien 1950 (zusammen mit einem von Bultmann selbst angefertigtes Ergänzungsheft); der Kommentar erlebte in dieser Form insgesamt zehn (unveränderte) Neuauflagen (zwischen 1952 und 1986 als 12. bis 21. Auflage).

57 Die drei Johannesbriefe (KEK xIII; Göttingen: Vandenhoeck \& Ruprecht, 1967 ${ }^{7}$ ).

58 Der zweite Brief an die Korinther (hg. E. Dinkler; KEK Sonderband; Göttingen: Vandenhoeck \& Ruprecht, 1976).

59 Zum Verhältnis von Bultmann zu Lohmeyer vgl. Hammann, Rudolf Bultmann. Eine Biographie (s. Anm. 52), 243f, der Differenz und Nähe von Bultmann zu Lohmeyer so beschreibt: Für Bultmann habe Lohmeyer erkannt, „daß es nicht ausreiche, religiöse Phänomene und Begriffe bloß aus ihrem geschichtlichen Kontext zu erklären, sondern dass sie allein , aus ihrem Ursprung in der Erfassung der menschlichen Existenz selbst' recht zu verstehen seien. Wie Lohmeyer diese Aufgabe - im Rekurs auf Gedanken seines philosophischen Gewährsmannes Richard Hönigswald - bearbeitete, hielt Bultmann weithin für verfehlt. Er schätzte seinen Nachfolger in Breslau [d.h. E. Lohmeyer] aber wie sonst keinen weiteren deutschsprachigen Neutestamentler seiner Generation gerade deshalb, weil er in seinem erstaunlich produktiven Werk die Selbstbeschränkung der Exegese auf einen historistischen Positivismus aufbrach und die systematisch-theologischen Implikationen der wissenschaftlichen Auslegung biblischer Texte im Blick behielt“ (243, das Zitat im Zitat stammt aus einer Besprechung einer Veröffentlichung Lohmeyers durch Bultmann).

60 Lohmeyer, Die Briefe an die Philipper, an die Kolosser und an Philemon (s. Anm. 53), $3 *$

61 Ebd.

62 Staatsbibliothek zu Berlin. Preußischer Kulturbesitz, Nachlass 494 (Archiv des Verlages Vandenhoeck \& Ruprecht) Autorenkorrespondenz G 1937-1970, Tasche 027, Bl. 496: Brief des Verlags Vandenhoeck \& Ruprecht an R. Bultmann vom 4.6.1941; für die großzügige 
Johanneskommentars gäbe es Leser, die auf die noch ausstehende Einleitung warteten. Bultmann verweigert eine solche Einleitung. Er schreibt an den Verlag:

Natürlich werden Leser, die den Inhalt des Kommentars kennen lernen möchten, ohne sich die Mühe der Durcharbeitung zu machen, eine zusammenfassende Einleitung vermissen. Aber ich lege keinen Wert darauf, ihnen entgegen $\mathrm{zu}$ kommen. ${ }^{63}$

Immerhin hat Bultmann auf Bitten des Verlags seinen Standpunkt in einem "Nachwort“, das als Einlegeblatt dem gebundenen Kommentar beigegeben wurde, erläutert: ${ }^{64}$

Ich habe darauf verzichtet, dem Kommentar eine Einleitung, wie es sonst üblich ist, voraufzuschicken. Was in ihr über die literargeschichtlichen und literarkritischen Fragen zu sagen wäre, ist im Kommentar, zumal in den Analysen, die der Exegese jeweils vorausgehen, hinreichend gesagt. Ebenso enthält der Kommentar selbst das Notwendige über den theologischen Gehalt und die religionsgeschichtliche Stellung des Evangeliums. Eine zusammenfassende Darstellung glaubte ich in diesen Hinsichten durch ausführliche Register ersetzen zu können. Was aber die Diskussion der Verfasserfrage betrifft, so trägt sie für die Exegese gar nichts aus und scheint mir deshalb nicht in einen Kommentar, sondern in das Gebiet der „Einleitung in das Neue Testament" bzw. in das der alten Kirchengeschichte zu gehören.

Marburg, im Juli 1941

Rudolf Bultmann.

Gleichzeitig gibt es auch einen auffälligen Unterschied zwischen Lohmeyers und Bultmanns Kommentaren. Lohmeyers Kommentar zum Philipperbrief (in der Separatausgabe von 1928) ist der erste Kommentar im KEK überhaupt, der eine Übersetzung enthält, die dem jeweils behandelten Textabschnitt vorangestellt ist. Eine derartige Übersetzung fehlte in allen bisherigen Kommentaren, angefangen vom ersten Kommentar 1832 von Meyer zu den Synoptikern, bis 1924, dem Kommentar von Windisch zum 2. Korintherbrief. Der Grund für dieses in der Tat ungewöhnliche Verfahren ist zunächst darin zu sehen, dass Meyer selbst als Auftakt seiner Kommentararbeit eine Text- und Übersetzungsausgabe des gesamten Neuen Testaments vorangeschickt hatte, so dass die einzelne Übersetzung

Überlassung der Fotokopien aus dem Archiv des Verlags (mit Datum vom 17.3.2017) danke ich dem zuständigen Referatsleiter Herrn Dr. Ralf Breslau sehr herzlich.

63 R. Bultmann in einem Schreiben an den Verlag vom 13.6.1941 (Staatsbibliothek Berlin (s. die vorige Anm.), Bl. 495).

64 Bultmann hat diesen Text allerdings nur unwillig verfasst. Er schreibt am 13.6.1941, „Ich sende Ihnen nun einliegend ein kurzes Nachwort und überlasse es Ihnen, ob sie es in der von Ihnen angegebenen Weise verwerten wollen. Ich selbst lege keinen besonderen Wert darauf. Die Leser meines Kommentars könnten ja auch durch eigene Ueberlegung schliesslich merken, dass der Kommentar im Grunde einer Einleitung in der üblichen Weise nicht bedarf.“ 
sich zunächst erübrigte. Dabei ist es bis 1928 geblieben. Hier hat der Verlag für eine Änderung gesorgt.

Lohmeyer schreibt im Vorwort zur Separatausgabe des Philipperkommentars von 1928: „Auf Wunsch des Verlages ist der Erklärung eine deutsche Übersetzung beigegeben“65 - dieses „auf Wunsch des Verlages“ hat er 1929 im Vorwort der Gesamtausgabe fortgelassen, ${ }^{66}$ aber es folgt jeweils eine Zusatzbemerkung, etwas unterschiedlich formuliert, die 1929 so lautet: „dass sie [nämlich die Übersetzung] den griechischen Text nicht ersetzen, sondern im strengen Sinne ihn übersetzen, d.h. zugleich deuten soll, ist vielleicht nicht ganz unnütz hervorzuheben“. ${ }^{67}$ Lohmeyer hat diese Möglichkeit der Textdeutung auf dem Wege der Übersetzung sofort beim Schopfe ergriffen, und zwar indem er wiederholt den übersetzten Text in Stichen anordnete, so dass das Druckbild bereits die in der Auslegung vorausgesetzte bzw. ermittelte Textstruktur erkennen ließ. Dies gilt neben anderen Teilen des Phil insbesondere für den sog. Philipperhymnus Phil 2,6-11. Hier macht schon die Druckanordnung Lohmeyers Strukturanalyse sichtbar. ${ }^{68}$ Analoges gilt dann für seinen Mk-Kommentar von 1937, wo er den Redentext von Mk 13, 5-37 in Stichen gesetzt hat. ${ }^{69}$

Bultmann hat sich dagegen konsequent geweigert, dem Wunsch des Verlages nach einer Übersetzung des kommentierten Texts nachzukommen. Schon 1928 schreibt er an den Verlag, offenbar auf eine entsprechende Anfrage: „Es wird vermutlich wichtiger sein, den griechischen Text in der stichischen Ordnung (in den in Frage kommenden Partien $)^{70}$ zu drucken, als eine Übersetzung ${ }^{\text {“ }}{ }^{71}$ Dabei ist es dann geblieben, und das gilt nicht nur für den 1941 abgeschlossenen Kommentar zum Johannesevangelium, sondern sogar noch für das Spätwerk, den Kommentar zu den Johannesbriefen von $1967 .^{72}$

65 Auf der Rückseite des Titels (Pappeinband), ohne Seitenzählung; bibliographische Angaben s. Anm. 53.

66 Lohmeyer, Die Briefe an die Philipper, an die Kolosser und an Philemon (s. Anm. 53), $3 *$

67 Ebd.

68 Lohmeyer, Die Briefe an die Philipper, an die Kolosser und an Philemon (s. Anm. 53), 72; andere in Stichen gesetzte Texte: Phil 1.1-2, 3-6, 27-30; 2.1-4.

69 Lohmeyer, Das Evangelium des Markus (s. Anm. 54), 269, 274f, 279f; eher verwunderlich ist die Anwendung dieses Verfahrens auf einen Erzähltext wie Mk 4.35-41 (a.a.O., 89).

70 Gemeint sind die Texte, die Bultmann der vorjoh Quelle der Offenbarungsreden zuschreibt, beginnend mit 1,1-5.9-12; Bultmann ist dann im Kommentar auch so verfahren, vgl. die Verweise im Register unter dem Stichwort: „Quellen: Offenbarungsreden“ im Kommentar auf S. 559.

71 Staatsbibliothek Berlin Preuß. Kulturbesitz, Nachlass 494 (Verlagsarchiv Vandenhoeck \& Ruprecht, Ordner 154 („Autoren 1928 A - E - D)“: Bultmann Rudolf; Brief vom 16.12.1928 (handschriftlich).

72 Die Übersetzung im KEK Sonderband: Der zweite Brief an die Korinther (s. Anm. 58) stammt vom Herausgeber Erich Dinkler! Vgl. E. Dinkler, „Vorwort und Einleitung des Herausgebers“, ebd., 9. 
Abbildung 5 zeigt ein Verlagsprospekt von 1937, eine „Subskriptionseinladung“",73 und zwar für insgesamt fünf Bände, deren Neubearbeitung vor kurzem erfolgt war, bzw. in Kürze erwartet wurde. Dabei sind zwei Kommentare bereits erschienen: E. Lohmeyer: „Das MarkusEvangelium" 1937 und O. Michel: „Der Hebräerbrief“75 bereits 1936. Besonders interessant sind die Angaben über die noch in Vorbereitung befindlichen Bände:

(a) R. Bultmann: „Das Johannes-Evangelium“; ${ }^{76}$ am Fuß der Seite heißt es, die erste Lieferung ist „vor kurzem erschienen“. Die siebte und letzte Lieferung erschien dann 1941, und Bultmanns Kommentar war der letzte, der geschlossen vor dem Ende des Zweiten Weltkriegs erschienen ist. ${ }^{77}$

(b) Aufschlussreich ist auch die Ankündigung des Kommentars über den Römerbrief, der ursprünglich von Ernst von Dobschütz verfasst werden sollte. Nach dessen Tod im Jahre 1934 war der aus Norwegen stammende, in Schweden lehrende Neutestamentler Anton Fridrichsen vorgesehen. Fridrichsen war mit der deutschen Theologie eng verbunden, mit Bultmann befreundet und 1927 Ehrendoktor der Theologischen Fakultät Marburg geworden. ${ }^{78}$ Allerdings: Nach dem deutschen Überfall auf

73 Vorlage: Privatbesitz des Verfassers. Der Titel ist in der deutschen Sütterlin-Schreibschrift gesetzt, der Rest des Prospekts in Fraktur. Zur Einführung des Fraktursatzes im KEK vgl. Koch, „Chronologie“ (s. Anm. 5), $476 f$.

74 Auf der Titelseite selbst heißt der Kommentar dagegen: „Das Evangelium des Markus“.

75 Auch hier besteht eine Differenz zum tatsächlichen Buchtitel; er lautet: „Der Brief an die Hebräer".

76 Der Titel von 1941 lautet: „Das Evangelium des Johannes“. Der Kommentar sollte ursprünglich der Universität St Andrews gewidmet sein, als Dank für die 1935 erfolgte Verleihung der theologischen Ehrendoktorwürde (vgl. Hammann, Rudolf Bultmann. Eine Biographie (s. Anm. 52), 475). Aufschlussreich ist Bultmanns Brief vom 4.11.1940 (Staatsbibliothek zu Berlin. Preußischer Kulturbesitz, Nachlass 494 (Archiv des Verlages Vandenhoeck \& Ruprecht) Autorenkorrespondenz G 1937-1970, Tasche 027, Bl. 509), in dem Bultmann auf Bedenken des Verlags gegenüber dieser Widmung (während des Krieges gegen Großbritannien!) antwortet: „Was die Widmung betrifft, so danke ich Ihnen für Ihren Brief vom 30. Sept. Ich habe daraufhin die Absicht, das Buch der Universität St. Andrews zu widmen, mit Bedauern fallen lassen und hoffe, dass ich ihr dann den Kommentar zu den Johannesbriefen widmen kann. Ich möchte nunmehr das Buch meinen alten Marburger Freunden und Arbeitsgenossen widmen. Es genügt dafür die Formel: ,Den alten Marburger Freunden'." Damit sind jene befreundeten jüdischen Kollegen gemeint, für die sich Bultmann selbstlos eingesetzt hat, vgl. Hammann, Rudolf Bultmann. Eine Biographie (s. Anm. 52), 283-9; zur Widmung: 298. Natürlich konnte Bultmann diese Freunde nicht namentlich nennen. Aber die Formulierung ist aufschlussreich genug.

77 Vgl. auch Ruprecht, „Der kritisch-exegetische Kommentar als Verlagsprojekt“ (s. Anm 17), 67f. 78 Vgl. Hammann, Rudolf Bultmann. Eine Biographie (s. Anm. 52), 244 f. 


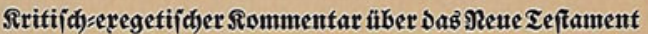
begrün Det von heintid If

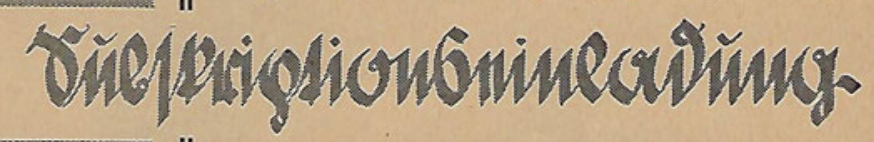

Utm Den alten, bewăbrfen Srommentar auf Der Ģ̄̆be Der modernen

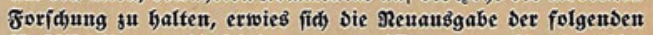
fünf Abteilungent, Die feit langem vergriffen waren, als notwendig.

\section{Das Diarł}

Bearbeitef won Prof. D. Ernft Bofmener

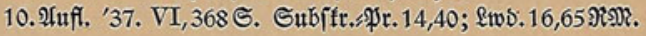

\section{Das So hames: Evangelium}

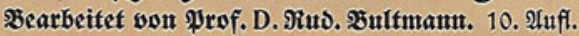

\section{Dex Römerbrief}

bearbeitet von Prof. D. (E. v. Dobifitik unt nad Deffen

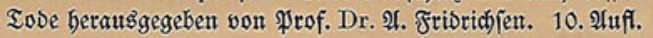

\section{Der Galatexbrief}

Searbeitet won Prof. Bic. Seintid Sd)lier. 10. Inff.

\section{Der Sebräerbrief}

ઝearbeitet won \$riwafbojent \&ic. \$fto Midjel

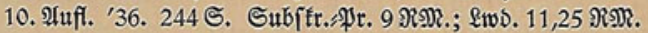

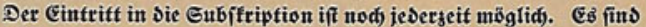

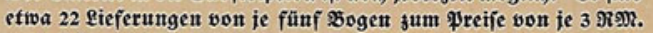

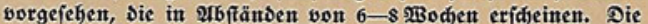
bereits vorliegenden Rieferungen bejw. \$ände tönnen in $\Re$ a ten bejaflt werben.

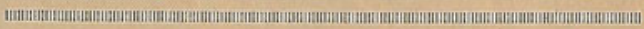

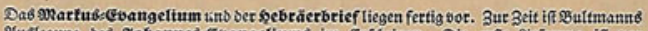

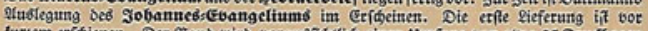

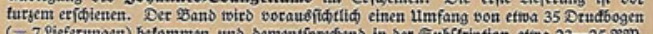

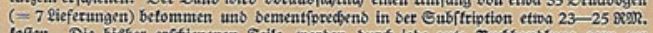

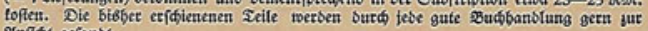
Dinfít gefandt.

Abbildung 5. Verlagsprospekt von 1937, S. 2: „Subskriptionseinladung“. 
Norwegen gab Fridrichsen diesen Auftrag zurück. ${ }^{79}$ Mit der Kommentierung des Römerbriefs wurde dann Otto Michel beauftragt. ${ }^{80}$

(c) Schließlich wird auch die Kommentierung des Galaterbriefs durch Heinrich Schlier angekündigt; die erste Lieferung konnte noch 1939 ausgegeben werden, der Gesamtkommentar erschien jedoch erst $1949 .{ }^{81}$

Bultmanns Kommentar konnte allerdings erst nach 1945 seine Wirkung entfalten. Er erschien 1950 in korrigierter Auflage, die dann noch zehnmal nachgedruckt wurde, analog zu Lohmeyers Mk-Auslegung von 1937, die 1951 postum in durchgesehener Auflage erschien und dann noch sechsmal nachgedruckt wurde. Und es gibt noch eine weitere Gemeinsamkeit: $\mathrm{Zu}$ beiden Kommentaren erschien mit der ersten korrigierten Auflage ein Beiheft mit größeren Ergänzungen und Änderungen, die jeweils auf die Autoren zurückgingen, bei Bultmann ohnehin und bei Lohmeyer postum aufgrund der Notizen in seinem Handexemplar. ${ }^{82}$

Ein Paukenschlag war dann der Apostelgeschichtskommentar von Ernst Haenchen; Haenchen war von Hause aus Systematiker, ab 1939 in Münster

79 Brief von Anton Fridrichsen vom 8.5.1940 an den Verlag Vandenhoeck \& Ruprecht (Staatsbibliothek zu Berlin. Preußischer Kulturbesitz, Nachl. 494 (Archiv des Verlages Vandenhoeck \& Ruprecht) G 1937-1970, Bl. 176): „Leider muss ich Ihnen mitteilen, dass meine Mitarbeiterschaft an dem Meyer'schen Kommentar nicht mehr in Frage kommen kann. Die Entwicklung der Zeitlage steht der wissenschaftlichen Wirksamkeit entgegen, und wir müssen uns hier im Norden auf andere Aufgaben konzentrieren." Zwar formuliert Fridrichsen nur in Andeutungen, aber der Hinweis auf die Zeitlage (aus der Perspektive des Nordens!) ist eindeutig: Der Brief datiert vom 8.5.1940, die Besetzung Norwegens durch Deutschland begann am 9.4.1940.

80 Der Kommentar erschien 1955: O. Michel, Der Brief an die Römer (KEK rv; Göttingen: Vandenhoeck \& Ruprecht, $1955^{10}$ ), XVI +357 Seiten; in seinem Vorwort wird der tatsächliche Hintergrund für Fridrichsens Verzicht von Michel verschleiert, wenn er schreibt: „Leider war es auch A. Fridrichsen ... im Laufe der Kriegswirren unmöglich, die Arbeit von E. v. Dobschütz zum Abschluß zu bringen; er erkannte, daß ein neuer Anfang gemacht werden müsse, und übergab mir im Frühjahr 1940 diese schwere Aufgabe." Dazu ist festzustellen: (a) Die nebelhaften „Kriegswirren“ lassen sich, wie der Brief von A. Fridrichsen zeigt (s. die vorige Anm.) präzise benennen, Michel umgeht sie jedoch; (b) Fridrichsen hatte in dem o. a. Brief geschrieben: „Für die Bearbeitung des Kommentars schlage ich vor Herrn Michel in Halle, der zweifellos den Auftrag mit Freuden übernimmt." Das klingt schon anders, und vor allem: Dass Fridrichsen den Kommentar (auch) deswegen abgegeben habe, weil ein Neuanfang durch einen anderen Kommentator notwendig sei, ist mit keinem Wort angedeutet. Das ist Selbststilisierung von Michel; vgl. dazu auch M. Theobald, „Der Römerbrief“, Der „Kritisch-exegetische Kommentar" in seiner Geschichte (s. Anm. 1), 184-208, hier 184f (mit Anm. 7).

81 H. Schlier, Der Brief an die Galater (KEK vII; Göttingen: Vandenhoeck \& Ruprecht, $1949^{10}$ ), $11^{*}$ +211 Seiten.

82 S.o. Anm. 54. 
tätig, aber bereits 1946 emeritiert. ${ }^{83}$ Der Kommentar, der 1956 erschien, ${ }^{84}$ gehört faktisch in die Phase der sich entwickelnden Redaktionsgeschichte, und fragt nicht nach den historischen Ereignissen, von denen Lk erzählt, sondern danach, was Lk seinen Lesern von diesen Ereignissen erzählt und wie er es erzählt. ${ }^{85}$ D.h. Haenchen dreht die Perspektive um und nimmt damit viele heutige Einsichten der narrativen Exegese vorweg. Haenchen hat unermüdlich an seinem Kommentar weitergearbeitet. Fünf weitere Auflagen sind erschienen, die letzte 1977 bereits postum, wobei drei Auflagen gründliche Umarbeitungen darstellen. ${ }^{86}$

Mit diesem „Klassiker“ zur Auslegung der Apostelgeschichte soll der Durchgang durch den ursprünglich von Meyer im ersten Drittel des 19. Jahrhunderts begründeten „Kritisch-exegetischen Kommentar über das Neue Testament" beendet werden. ${ }^{87}$ Die Tendenzen des 20. Jh.s setzen sich im 21. Jh. fort. Folgende Grundtendenzen sind erkennbar:

1 Die philologische, religionsgeschichtliche und traditionsgeschichtliche Analyse der zu kommentierenden Texte ist unumstritten.

2 Genauso unabweisbar ist, dass die Texte als theologische Texte ernst zu nehmen sind, was in der jeweiligen Interpretation zur Geltung kommen sollte.

3 Der Selbstanspruch der Kommentarreihe ist dabei ein doppelter:

a. Der Kommentar will den Lesern eine Analyse und Interpretation der jeweiligen neutestamentlichen Schrift auf der Höhe der gegenwärtigen Fachdiskussion liefern.

$83 \mathrm{Zu}$ E. Haenchen gibt es faktisch keine biographische Darstellung; vgl. daher ersatzweise D.-A. Koch, „Ernst Haenchen“, Der „Kritisch-exegetische Kommentar“ in seiner Geschichte (s. Anm. 1), $510 f$.

84 E. Haenchen, Die Apostelgeschichte (KEK III; Göttingen: Vandenhoeck \& Ruprecht, $1956^{10}$ ), $12^{*}$ +665 Seiten.

85 Zur Würdigung von Haenchens Kommentar vgl. D. Marguerat, „Die Apostelgeschichte“, Der „Kritisch-exegetische Kommentar“ in seiner Geschichte (s. Anm. 1), 161-83, hier 162: „Ohne Übertreibung wird von einer Actaforschung vor Haenchen und einer Actaforschung nach Haenchen gesprochen."

86 Die 11. Auflage von 1957 (ist ein reiner Nachdruck), die 12. Auflage von $1959\left(16^{*}+661\right.$ Seiten), die 13. Auflage von $1961\left(16^{*}+694\right.$ Seiten) und die 14. Auflage von $1965\left(16^{*}+694\right.$ Seiten) enthalten z.T. erhebliche Überarbeitung; die Ausgabe von 1965 wurde als 15. Auflage 1968 nachgedruckt, und die umfangreiche 16. Auflage (die 7. der von Haenchen besorgten Auflagen) erschien mit einem Vorwort von Erich Gräßer postum 1977 (717 Seiten).

87 Hinzuweisen ist auch auf die in Großbritannien bzw. den USA erschienenen Übersetzungen von insgesamt neun Kommentaren in der Zeit zwischen 1971 und 1996 ins Englische; es handelt sich (in alphabetischer Reihenfolge) um R. Bultmann (JohEv, 2 Kor, $1-3$ JohBr), H. Conzelmann (1 Kor), M. Dibelius (Jak), L. Goppelt (1 Petr), E. Haenchen (Apg), E. Lohse (Kol, Phlm), G. Strecker (1-3 JohBr); genau Aufstellung s. bei Koch, „Chronologie“ (s. Anm. 5), $503 f$. 
b. Der Kommentar will den Lesern eine Auslegung bieten, die auch in 20 oder 30 Jahren noch Bestand hat und nicht nach fünf Jahren überholt ist.

4 Ob allerdings ein Kommentar ein epochemachendes Werk sein wird oder nicht (und es gab ja im KEK durchaus echte Klassiker!), das lässt sich nicht im Voraus festlegen und daher auch in keinen Verlagsvertrag hineinschreiben.

German abstract: Der „Kritisch-exegetische Kommentar über das Neue Testament" (früher: Der „Meyer") ist vor über 180 Jahren, nämlich 1829 von $\mathrm{H}$. A. W. Meyer begründet worden. Er ist somit in Deutschland (und darüber hinaus) die älteste noch bestehende Kommentarreihe zum Neuen Testament und stellt daher ein interessantes forschungsgeschichtliches Untersuchungsfeld dar. Schlaglichtartig werden die Entwicklungen der ntl. Wissenschaft, der Wechsel der Perspektiven, Methoden und Schwerpunkte, aber auch konstante Faktoren erkennbar. Bemerkenswert ist darüber hinaus, in welchen Phasen der Kommentar durch Übersetzungen auch im englischsprachigen Raum rezipiert wurde - und zugleich wie stark auch die zeitgeschichtlichen Rahmenbedingungen, insbesondere die Umbrüche im 2o.Jh., die Geschichte des Kommentars beeinflussten. 